\title{
Social Media Live Streaming y su impacto en los medios de comunicación: experiencias editoriales en América Latina
}

\author{
doi: 10.33264/rpa.202101-02 \\ Alexis Apablaza-Campos \\ Facultad de Humanidades y Ciencias Sociales, Universidad UNIACC \\ Lluís Codina ${ }^{3}$ \\ Departamento de Comunicación, Universitat Pompeu Fabra
}

\section{Resumen}

Pese a que el video en directo en formato digital existe desde hace más de una década, este solo encontró su maduración cuando se integró a las redes sociales a partir de 2015, tecnología conocida como Social Media Live Streaming (SMLS). El periodismo, con especial énfasis en los cibermedios, ha aprovechado esta innovación para buscar nuevas fórmulas que permitan tanto interactuar como fidelizar a sus audiencias.

El presente artículo caracteriza los canales SMLS en medios de comunicación de América Latina, buscando comprender su rol con relación de las tendencias del periodismo digital. Adicionalmente, este trabajo presenta un acercamiento al empleo de esta tecnología en diversos medios en español incluyendo un análisis de caso sobre el uso del live streaming (Periscope y Facebook Live) de NTN24 Venezuela durante las Elecciones Regionales de 2017.

Los resultados permiten concluir que los editores de diversos medios de comunicación latinoamericanos ya cuentan con amplia experiencia en emisiones SMLS, incluyendo algunos casos de éxito destacados a nivel mundial por las propias redes sociales. Finalmente, se detallan los motivos que hacen compleja la posibilidad de realizar un análisis comparativo que permita comprender con suficiente claridad qué canal Social Media Live Streaming puede ofrecer mejores resultados a sus editores de contenido.

Palabras clave: Social Media Live Streaming, SMLS, medios digitales, cibermedios, Periscope y Facebook Live.

\section{Abstract}

Although live video in digital format has existed for more than a decade, it only found its maturity when it was integrated into social networks from 2015, technology known as Social Media Live Streaming (SMLS). Journalism, with special emphasis on news outlet, has taken advantage of this innovation to find new formulas that allow

\footnotetext{
${ }^{3}$ Esta investigación cuenta con el apoyo de CONICYT (Comisión Nacional Científica y Tecnológica de Chile), a través del programa "Becas Chile" Doctorado en el Extranjero, número de folio 72170334 correspondiente a Alexis Apablaza-Campos. Asimismo, este trabajo forma parte del proyecto "Creación y contenido interactivo en la comunicación de información audiovisual: audiencias, diseño, sistemas y formatos". Ref: CSO2015-64955-C4-2-R (MINECO/FEDER), Ministerio de Economía y Competitividad (España).
} 
both interacting and loyalty to its audiences.

This paper characterizes the SMLS channels in Latin American media, seeking to understand their role in relation to the trends of digital journalism. Additionally, this work presents an approach to the use of this technology in various Spanish news media including a case study on the use of live streaming (Periscope and Facebook Live) of NTN24 Venezuela during the 2017 Regional Elections.

The results allow us to conclude that the editors of various Latin American media already have extensive experience in SMLS broadcasts, including some success stories highlighted worldwide by the social networks themselves. Finally, the reasons that make the possibility of carrying out a comparative analysis that allows us to understand with sufficient clarity which Social Media Live Streaming channel can offer better results to its content publishers are detailed.

Keywords: Social Media Live Streaming, SMLS, digital news media, news outlet, Periscope and Facebook Live.

\section{Introducción}

\section{Antecedentes}

La llegada del siglo XXI trajo, entre otras muchas cosas, algo de caos y tormenta a Internet y, de paso a los entornos digitales. Días previos al atentado a las Torres Gemelas del 11/09/2001, la crisis de la burbuja.com demostró que el intento del mercado de rentabilizar la web llevó a la sobreespeculación y, por consiguiente, a la bancarrota a diversas startups de todo el mundo.

La cuestión es que Internet se vio obligada a repensarse a sí misma antes de que se cumpliese la primera década de la implementación de la World Wide Web. Es por ello que nacen conceptos como la Web 2.0, que se enfocaba en dos aspectos que después han tenido una influencia duradera.

En primer lugar, aportó el enorme cambio que han significado los contenidos generados por los usuarios. Realmente, es imposible exagerar la importancia que esto ha tenido para el ecosistema de la comunicación digital, ya que ha aportado un cambio, posiblemente, de forma permanente, a toda la comunicación social.

Gigantes como Wikipedia y YouTube proceden de esta herencia. Pero también las propias redes sociales, que deben su enorme implantación social al impulso inicial que supusieron los contenidos generados por los usuarios, por más que ahora, los medios de comunicación también las hayan colonizado.

El segundo componente, no menos influyente, fue la aparición de la computación en la nube y la vinculación de tales aplicaciones entre sí. De aquí, apareció un nuevo entorno gracias al cual, una amplia gama de apps (aplicaciones móviles) estaba en condiciones de conectarse entre sí y aportar de este modo, una nueva capa de 
conectividad y de interactividad a los usuarios, vía redes sociales, por ejemplo, pero también de interactividad entre las propias aplicaciones, entre robots, en definitiva.

\section{El caso del video digital}

Es en medio de este camino cuando el video digital sufre una transformación radical tal cómo se le había conocido hasta ese momento. Fue durante el San Valentín de 2005, concretamente, cuando YouTube vio la luz con un sistema de reproducción de contenidos multimedia acorde a la velocidad de conexión del usuario.

Tras 20 meses de su lanzamiento, la plataforma de videos en línea fue comprada por Google en US\$1.650 millones; y en 2006, cuando la revista Time eligió a "You" como personaje del año para destacar la importancia de los contenidos generados por los usuarios en pleno auge de la denominada "era global de la información" destacando en su portada un ordenador que en su pantalla contiene un video de YouTube. Así lo informó La Nación de Costa Rica “Time considera que YouTube cambió las reglas del intercambio de información porque, en los últimos doce meses, miles de personas se han vuelto famosas, queriéndolo o no, con un video puesto en este sitio electrónico" (Nación, 2006).

A tal punto llegó esta revolución en la forma de ver videos en línea que muchos usuarios comenzaron espontáneamente a subir contenidos de canales de televisión -tanto históricos como actuales- convirtiendo rápidamente a la plataforma en la videoteca digital más grande de la historia. Todo ello conllevó a un cambio de paradigma para los televidentes: "si anoche no pude ver mi programa favorito, no importa. Mañana lo busco en YouTube”. (Apablaza Campos A. , 2017, p. 149)

Pese a que ante la más mínima denuncia de violación de contenidos de autor la plataforma de Google retira los videos, ello no libró a YouTube de largas batallas legales con grandes empresas de medios -como Viacom-, las cuales, tras años y años, simplemente terminaron en acuerdos extrajudiciales aplicando la lógica del "si no puedes vencerles, únete a ellos": actualmente estas cadenas tienen su canal oficial en YouTube y así pueden controlar la difusión y reproducción de sus contenidos.

La experiencia que los medios de comunicación obtuvieron con este proceso fue que, como mínimo, debían ofrecer de una plataforma de videos en su sitio web como parte de sus estrategias de difusión de contenidos.

Netflix, en ese entonces una incipiente empresa de venta de DVDs a domicilio, observó atentamente ese fenómeno y se demoró alrededor de una década en convertirse en un servicio de alquiler de series y películas en línea. Como consecuencia, solo en 2019 obtuvo una facturación superior a los US\$20.150 millones y un total de 170 millones de suscriptores en todo el mundo (Dircomfidencial, 2020). 
Así, la segunda década del nuevo siglo ofrece mayor contenido, y mayores oportunidades de consumo, de videos en línea. Esto va de la mano con conexiones a internet de mayor calidad y en distintos formatos, especialmente en Entornos Móviles, según explica el director de la Escuela de Comunicación Digital de la Universidad del Pacífico de Chile, Guillermo Bustamante, a El Economista:

Ahora el mundo es en tiempo real, nos enteramos casi inmediatamente de lo que está sucediendo... La conectividad cada vez es mayor y el video genera más compromiso en las personas. Tiene menos barreras de entrada, es más visual y es más práctico (El Economista América, 2016).

\section{Cambios en el ecosistema: el SMLS y sus implicaciones}

En 2007, casi en paralelo al crecimiento de YouTube y Netflix, nace Justin.tv como una web que permite la emisión de videos en directo. Sin embargo, es absorbida siete años más tarde por Twitch, servicio de streaming para videojuegos que pertenece a Amazon.

Al año siguiente, en 2015, aparece Merkaat como una aplicación móvil que permite la emisión de videos en directo y compartirlos en redes sociales. Llegó a obtener dos millones de usuarios y fue ampliamente aplaudida tanto por medios de comunicación como por diversos trabajos académicos hasta que, sin mediar aviso, desapareció de las apps stores y fue descontinuada en octubre de 2016 (Mlot, 2016).

Estos fracasos no fueron impedimento para que Twitter adquiriese Periscope a principios de 2015, cuando solo era una app en desarrollo, por US\$100 millones y decidiese lanzarla al mercado en marzo del mismo año.

¿Cómo se puede entender esta decisión? Nosotros entendemos que, la razón se debe a que el Social Media Live Streaming (SMLS) ${ }^{4}$ aporta a los usuarios mucho más que video en directo.

En particular, y de acuerdo con nuestros análisis, entendemos que, para que una tecnología sea considerada como SMLS debe cumplir con dos siguientes características, ambas muy importantes:

1. Realizar y distribuir vídeos en directo, en diferentes formatos, a través de las redes sociales, mediante aplicaciones propias asociadas a las mismas.

2. Proporcionar al emisor un feedback instantáneo: número de usuarios conectados, mensajes y emoticones de sus audiencias. (Apablaza-Campos, 2018a, pág. 106).

\footnotetext{
${ }^{4}$ Desde aquí en adelante los términos Social Media Live streaming y SMLS se usarán como sinónimos de manera indiscriminada. 
El primer punto es muy importante porque implica unas facilidades de producción de video aún mayores que las preexistentes, que ya eran muy altas. Efectivamente, al poder utilizar las mismas redes que permiten la comunicación para la producción de contenidos que incluyan imagen y video en lugar de simple texto, tuvo lugar un nuevo escenario en estos entornos.

Con todo, en el segundo punto es posible encontrar otro valor diferencial, que tal vez pueda ser considerado como el más importante, la interacción. De hecho, la opción de intercambiar los roles de emisor y receptor en el proceso comunicativo permiten que el usuario perciba su participación en el contenido del video en directo y al mismo tiempo permite al comunicador tomar decisiones en simultáneo que faciliten el cumplimiento de las expectativas de sus audiencias y los objetivos de su medio de comunicación.

Este proceso, explicado por diversos especialistas, solo es comparable con lo que sucedía en la década del 90 con los estelares de televisión de amplios presupuestos, cuyo equipo de producción -al conocer la medición de sintonía al instantemodificaba continuamente la estructura de contenidos e invitaba a la interacción a través de concursos telefónicos. En todo caso, para acabar de situar al SMLS parece oportuno considerar la perspectiva que proponen dos trabajos recientes:[EI SMLS es] "un sistema escalable que involucra al emisor y al receptor en cada retransmisión: no hay usuarios pasivos, ya que incluso el espectador está interactuando en tiempo real con la escena" (Ossorio Vega, 2015). En la era del social media, el live streaming ayuda (a los emisores) a forjar su propia marca personal a la vez que hacen frente a las exigencias de la audiencia... No hay excusa para no estar constantemente retransmitiendo (Rugg \& Burroughs, 2016, p. 67).

\section{SMLS: canales y utilización}

Pese a que Periscope, como aplicación SMLS, fue descontinuada el 31 de marzo de 2021 (Periscope, 2020), sus funciones fueron integradas totalmente a Twitter, por lo cual la red social de videos en directo se ha convertido en la funcionalidad de perfiles SMLS de la red social de microblogging (Twitter, s.f.) $)^{5}$. Algo similar a lo que ya sucede con Facebook y Facebook Live.

Además de Periscope/Twitter y de Facebook Live/Facebook, existen una serie de recursos para emitir videos en directo a través de las redes sociales, que consideramos a continuación:

\footnotetext{
5Las emisiones SMLS de Periscope actualmente son consideras como emisiones SMLS de Twitter debido a que la API pública de la exred social de SMLS migró hacia la API de la red social de microblogging durante los primeros meses de 2021. Es por ello que los análisis relacionados con emisiones realizadas en Periscope siguen teniendo amplia vigencia y relevancia. Más información en https://help.twitter.com/es/using-twitter/periscope-faq 
- YouTube Live: todo usuario con cuenta Gmail puede emitir en directo desde un computador o un portátil previa habilitación de la funcionalidad (YouTube, s.f.).

- Instagram Stories en directo: tanto la emisión como la reproducción de los contenidos solo puede hacerse a través de dispositivos móviles y en formato vertical. También permite aplicar una serie de filtros en los rostros tanto antes de la transmisión como durante ella (Instagram, 2020).

- Linkedin Live: función disponible tanto para perfiles como páginas de organización. Al igual que YouTube Live requiere solicitud a la plataforma, la cual prioriza a usuarios que previamente hayan compartido contenidos en video a través de la red social de networking (LinkedIn, s.f.).

- TikTok Live: la red social de videos cortos permite a los usuarios y creadores de contenido mayores de 16 años interactuar entre ellos en tiempo real. Actualmente esta función solo está disponible en algunos países (TikTok, s.f.)

- SMLS propias de medios digitales: mientras la agencia Reuters anunció el desarrollo de Reuters Connect para canales de televisión y editores de video profesionales, la BBC confirmó que está trabajando en un servicio propio de live streaming para la difusión de sus contenidos (Thompson Reuters, 2017).

Un caso aparte y digno de destacar es el de China, país que cuenta con 280 millones de usuarios de banda ancha, 950 con conexiones móviles a través del sistema $4 \mathrm{G}$ y un consumo de datos promedio mensual de 2 GB por usuario (Xinhua, 2017). El Knight Center de la Universidad de Texas analizó los recursos que dispone el videoperiodismo en el gigante asiático.

En China, [donde no están presentes Facebook Live, Periscope y YouTube Live, pero cuentan con más 80 apps de SMLS] básicamente algunas están tratando de ser dominantes en el terreno de las plataformas de streaming en vivo, no sólo tener funciones de transmisión en vivo... Estoy seguro que la mitad de ellas morirán al final de este año o el próximo, Wang Jiapeng, de Caixin ${ }^{6}$ (Nazario, 2017).

No obstante, cabe señalar que gracias a la aparición de Periscope comenzó la era del Social Media Live Streaming tal como la conocemos ahora. En pleno lanzamiento, Kayvon Beykpour, creador de la app, explicó su razón de ser: "quisimos crear lo más parecido a la teletransportación".

Los buenos resultados llegaron rápido: 10 millones de descargas y 2 millones de usuarios activos en sus primeros cuatro meses y distinguida como "la app del 2015

${ }^{6}$ Uno de los principales medios financieros de China. Tiene una versión en inglés y en mandarín en http://www.caixinglobal.com/ 
para iPhones" por Apple (Publimetro Chile, 2016).

Actualmente, Periscope está totalmente integrada con su matriz Twitter, por lo cual basta con un tuit para emitir un vídeo en directo a través de ambas redes sociales. También dispone de diversos recursos que ya hemos mencionado en otros trabajos, pero que resulta oportuno reseñar:

- $\quad$ Periscope Producer: permite crear contenidos con una calidad más profesional en diferentes formatos como emisiones en $4 \mathrm{~K}$ o en realidad aumentada.

- $\quad$ Live 360: videos inmersivos en directo para quienes dispongan de los recursos adecuados para la emisión.

- $\quad$ AR Selfie Mask: el uso de filtros en rostros -tan comunes en redes como Snapchat e Instagram- para aplicar la realidad aumentada en directo.

- $\quad$ Anuncios pre-roll: a través de su programa Amplify se pondrá en marcha durante los próximos meses la primera experiencia con SMLS ADS7.

- Super Hearts: "supercorazones" que el consumidor de video en directo envía para contactar directamente con el emisor. Al ser una opción pagada, genera recursos para el 'periscoper'8.

- $\quad$ SMLS glass: gafas de sol, creadas por la marca Hawkers, que permiten la emisión de contenido en directo. (Pastor, 2017).

Facebook, por su parte, decide sumarse a la carrera del video en directo de una forma diferente. No crea una red social propia, sino que lo incluye como una funcionalidad para los perfiles, tanto del propio Facebook como de Instagram y de Facebook Mentions -creado para influencers y grandes marcas-. Su implementación fue paulatina hasta que abril de 2017 anunciaron que todo usuario con un perfil puede acceder a Facebook Live desde cualquier dispositivo.

Ante la situación de predominio actual de esta red, cabría preguntarse: ¿cómo es posible llegar de los últimos a la competencia por dominar el SMLS y rápidamente convertirse en el líder? La respuesta es simple: siendo Facebook.

Un reportaje de The Wall Street Journal informó acerca de los US\$50 millones que pagó la red social a diferentes medios digitales, marcas e influencers para que usaran su plataforma de video en directo como se puede ver en la Figura 1.

\footnotetext{
${ }^{7}$ Métodos de publicidad especialmente creados para su difusión a través de tecnologías compatibles con el Social Media Live streaming.

${ }^{8}$ Influencer en redes sociales que emite contenidos en directo. Más información en http://smediabusiness.com/periscoperun-nuevo-termino 
Más allá de las cifras, es importante destacar que Facebook es la tercera web con más visitas del mundo (Alexa, 2017). Esta es una importante ventaja competitiva para Facebook Live porque desde hace años que medios, marcas e influencers disponen de fanpages en su red social, así la emisión de un video en directo no les obliga ni a administradores ni a usuarios a mudarse a una red nueva -con todo el proceso que requiere la creación de un nuevo perfil- para seguir este contenido, sino que basta con recibir una notificación para ser invitar a sus seguidores.

Actualmente, Facebook está desarrollando programas que permitan mayores experiencias SMLS a través de diversos formatos, tales como videos inmersivos $/ 360^{\circ}$ (Live 360 Ready Program), realidad virtual (Oculus Rift) y realidad aumentada (AR Studio), todos ellos, qué duda cabe, de enorme interés para la evolución futura de los medios de comunicación.

También está testeando la opción de desarrollar un software que permita las transmisiones desde múltiples cámaras llamado Live Video Producer (López, 2017).

Por todo esto, no es de sorprender que el creador de Facebook, Mark Zuckerberg, anunciase en 2016 que "el avance más rápido de los próximos cinco años será principalmente en el vídeo" (Ingram, 2016).

Figura 1. Infografía sobre la inversión realizada por Facebook Live.

\section{Now Playing, Live on Facebook}

A selection of companies and individuals paid by Facebook to provide live video content. Amount of contracts, in millions of dollars

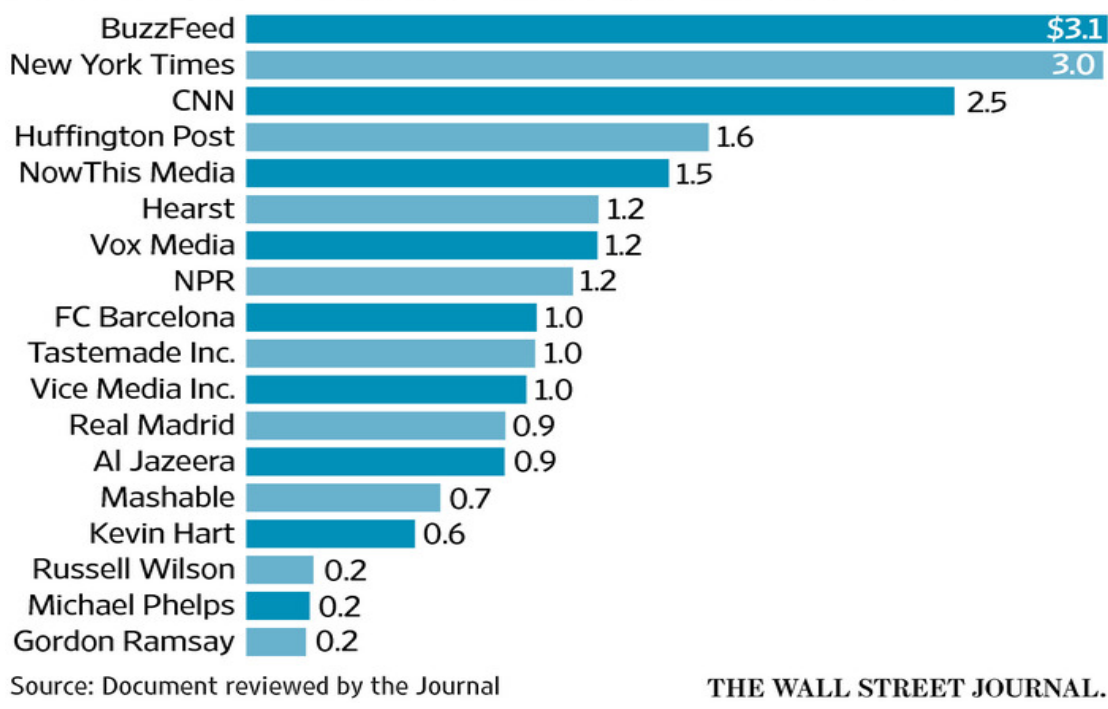

Fuente: Perlberg \& Sheetharaman (2016).

\section{SMLS y medios de comunicación}

Ante esta situación, cabe preguntarse: ¿cuál es la participación que han llevado a cabo los medios de comunicación en este tipo de contenidos? 
Una de las posibles respuestas podría estar en el informe Digital News Project 2017 (Newman, 2017a) promovido y publicado por el prestigioso Reuters Institute for the Study of Journalism de la Universidad de Oxford que asegura que este es "el año del video social y en directo" para coberturas informativas.

De hecho, según el estudio: "estos desarrollos son tanto para usar del poder del video para captar más atención-y, con ello, más publicidad-, como para combinar nuevas formas de expresión con un creciente interés en 'lo que está sucediendo ahora mismo". (Newman, 2017a, p. 5) La cuestión es que fue en abril del 2015 cuando por primera vez un periodista emplea las tecnologías SMLS durante una cobertura periodística. Fue Paul Levis, de The Guardian, quien emitió un video en directo desde su móvil a través de Periscope una manifestación contra la violencia policial sobre ciudadanos afroamericanos en las calles de Baltimore, Estados Unidos.

Meses más tarde destacó el caso de Paul Ronzheimer, redactor de Bild, quien fue enviado por el periódico alemán a la isla griega de Kos para informar cómo los refugiados sirios intentaban llegar hasta tierras bávaras. Sus emisiones en directo, durante los meses de agosto y septiembre del mismo año, no fueron tarea sencilla, ya que tuvo que cruzar las fronteras como si fuese un indocumentado más.

Durante las primeras semanas del año, se realiza en Las Vegas el evento CES, donde las principales empresas de tecnología exponen los mejores productos que están prontos a salir al mercado. Para la cobertura informativa durante la versión de enero 2016 del CES, el medio especializado Xataka entregó a sus enviados especiales dispositivos móviles exclusivos para la emisión de SMLS en su cuenta oficial de Periscope.

En cada emisión, los periodistas mostraban los diferentes dispositivos disponibles para que la comunidad de espectadores solicitara, mediante el chat de mensajes, qué prototipo querían conocer. Así la transmisión permitía a los usuarios conectados de cualquier parte del mundo vivir la experiencia como si estuviesen allí, cumpliéndose la deseada teletransportación citada por Beykpour. El uso de los SMLS de los medios de comunicación ha evolucionado en cuestión de meses desde la decisión espontánea del reportero de sacar su móvil y emitir en directo porque la calidad de la noticia lo amerita hasta convertirse en toda una estrategia digital de las redacciones. Una prueba de ello es el caso de The Washington Post, donde existe la figura del Facebook Live Editor, un departamento completamente al área digital del periódico. Su responsable es TJ Ortenzi, quien reconoce que cuenta con un equipo de cuatro profesionales que disponen de dos dispositivos móviles en cada salida a terreno: uno para contactarse con la redacción y otro exclusivo para la emisión del video en directo (Nelson, 2017).

En todo caso, y de acuerdo con nuestros análisis de comportamiento y la generación Revista Pensamiento Académico de la Universidad UNIACC 
de contenidos de los medios digitales, consideramos que es posible categorizar los usos fundamentales del periodismo en relación con las emisiones de Social Media Live Streaming, en cuatro grandes apartados:

1. Conferencias/ruedas de prensa

2. Sucesos de última hora

3. Backstage de programas

4. Emisión de señal televisiva

\section{Presentación y componentes del estudio}

El trabajo que presentamos en este capítulo utiliza un marco teórico compuesto por tres líneas principales:

\section{Periodística}

Entendemos la Periodística (también conocida como Teoría del Periodismo) como el estudio de las diferentes características y procesos que determinan la producción de contenidos de actualidad. Ella define el consumo actual de medios de comunicación como "un círculo de realidad envolvente que se convierte en referencia diaria de nuestra vida" (Gomis, 1991, p. 13). Consideramos que, en concreto, la así llamada Newsonomics (contracción de news y economics) forma parte de este apartado, debido a que analiza el conjunto de leyes que rigen tanto la selección de contenidos informativos como los resultados financieros de las empresas periodísticas (Doctor, 2010).

\section{Ecología de los Medios}

Cada vez tiene menos sentido analizar canales o medios de comunicación fuera de su contexto global y de las interacciones con otros medios y canales. De aquí la necesidad de incluir la ecología de los medios como uno de los componentes de este apartado. Gracias a la influencia de Marshall McLuhan, Neil Postman desarrolló esta teoría que plantea principalmente que "ningún medio adquiere sentido o existencia de manera aislada, sino en constante interrelación con otros medios". (Scolari, 2015, p. 18)

\section{Cibermedios}

A estas alturas, existe un amplio corpus teórico sobre cibermedios, entendidos como medios de comunicación digitales. Este corpus ha aportado desde modelos hasta procedimientos de análisis, pasando por un rico bagaje conceptual. Todo ello, será incorporado y tenido en cuenta como uno de los componentes de este marco.

Con la multimedialidad, hipertextualidad e interactividad como principales características, los cibermedios se definen como "aquellos sitios web cuya finalidad esencial es el desarrollo y transmisión de contenidos informativos, esto es, aquellos sitios web que cumplen en internet el papel de los medios de comunicación social" (Palacios \& Díaz Noci, 2007, p. 21). 


\section{Metodología}

En primer lugar, para el presente capítulo hemos llevado a cabo una revisión sistematizada sobre las publicaciones vinculadas con el tema central, con el fin de establecer un estado de la cuestión en este ámbito y relacionarlo con las disciplinas más próximas, algunas de las cuales ya hemos señalado, como cibermedios, redes sociales y visibilidad de las informaciones periodísticas. En segundo lugar, hemos llevado a cabo un análisis heurístico para analizar perfiles SMLS determinados, aplicando una serie de indicadores (KPI) que permitan medir la efectividad de estos canales. A partir de lo anterior, hemos llevado a cabo análisis comparativos que nos han permitido iniciar una línea de propuestas que, eventualmente, pueden ayudar a determinar el camino hacia el cual deben evolucionar los contenidos de videos en directo vinculándose con las expectativas de sus públicos objetivos.

\section{Objeto de estudio y objetivos}

El objeto de estudio de este trabajo, son los canales de SMLS de medios de comunicación latinoamericanos con énfasis en Periscope y Facebook Live e incluyendo un análisis de caso del cibermedio NTN24 Venezuela durante las Elecciones Regionales de 2017. Por su parte, el objetivo principal consiste en describir experiencias SMLS en medios de comunicación latinoamericanos, buscando comprender su posible rol en relación a las tendencias para el periodismo digital. Los objetivos derivados son los siguientes:

- Identificar las características de la integración del SMLS en medios digitales latinoamericanos como herramienta de distribución de información y uso en las coberturas periodísticas.

- Analizar los posibles impactos y resultados de medios digitales de la región que utilizan la red como su principal canal de distribución y emisión de videos en directo.

Con el fin de poder abordar los objetivos señalados, nos hemos planteado las siguientes preguntas de investigación:

- ¿Es posible determinar cómo los videos en directo son parte de la estrategia de contenidos de diversos medios digitales latinoamericanos?

- ¿Qué estrategias de contenidos han aplicado periodistas y editores latinoamericanos en la red social de SMLS que les ha permitido obtener mayor visibilidad a sus coberturas informativas? 


\section{Estudio y resultados}

\section{El SMLS en cibermedios latinoamericanos}

El informe Periodismo Innovador en América Latina, realizado por el ya mencionado Centro Knight de la Universidad de Texas, (Knight Center for Journalism in the Americas, 2017) contiene un análisis de coberturas periodísticas a través de Facebook Live de cinco medios digitales latinoamericanos e hispanohablantes realizadas durante el 24 de abril de 2016 y el 24 de enero de 2017. Cada pieza estudiada incluye el tipo de cobertura informativa, la fecha de la emisión, el alcance obtenido, la duración del contenido, el equipo humano y técnico requerido, y las lecciones aprendidas por los editores de cada medio para mejorar futuras emisiones. En publicaciones anteriores (Apablaza-Campos, 2018a; Apablaza-Campos \& Codina, 2018 b), realizamos un análisis adicional a los resultados obtenidos en el informe que aportó tres ideas principales:

- $\quad$ Salvo cuando se replicó por el SMLS la señal televisiva convencional o imágenes recibidas vía satélite, el equipo técnico empleado siempre fue un teléfono móvil inteligente.

- De las coberturas realizadas exclusivamente con un Smartphone, la mitad de ellas contó con, al menos, un camarógrafo y un reportero.

- Debido al desconocimiento de que las emisiones de Facebook Live tienen un límite, Univisión se vio obligada a establecer una alianza con la red social para evitar otra bajada repentina de la señal. (Apablaza-Campos, 2018a, pág. 116).

Asimismo, en la Figura 2 es posible ver el promedio de videos generados para cada cobertura informativa, las personas alcanzadas en promedio de las publicaciones analizadas y la duración promedio de las emisiones. Así los cinco medios analizados Univisión de Estados Unidos, Reforma y Animal Político de México, La República de Perú, y El Mostrador de Chile- obtuvieron una media de 98 usuarios alcanzados por cada segundo de emisión.

Figura 2. Promedio de vídeos, alcance y duración de emisiones SMLS

$\begin{array}{ll}\text { Promedio de videos por cobertura informativa } & 2,4 \\ \text { Alcance promedio obtenido } & 488.994 \\ \text { Duración promedio de las emisiones } & 1: 23: 16\end{array}$

Fuente: (Apablaza-Campos, 2018a, pág. 116)

En este informe, además, diversos editores de medios de comunicación latinoamericanos explican cuáles son los motivos por los cuales prefieren la generación de contenidos SMLS en Facebook Live. Crecimiento estable del alcance 
orgánico, información más detallada de quién pudo visualizar el vídeo, seguimiento de hashtags, y mayor masividad.

“Los números con Periscope fueron buenos, pero después sí sucumbimos a pasarnos a Facebook Live por una cuestión de masividad. Facebook es el lugar masivo donde está la gente. Más allá de que a los periodistas nos guste más Twitter, la gente está en Facebook y punto, y hay que producir donde la gente está". Máximo Tell, de Cadena 3 (Argentina). (Knight Center for Journalism in the Americas, 2017, p. 4-5).

A través del Facebook Journalism Project es posible conocer cuáles son las coberturas informativas exitosas en Facebook Live. En ellas, hay un caso latinoamericano, el de Teletrece ${ }^{910}$ de Chile, del cual se destacan las coberturas realizadas a los eventos deportivos como la Copa América Centenario y a la Eurocopa de Francia 2016. El 26 de junio de 2016 la Selección Chilena de Fútbol conquistó el título de la Copa América Centenario, y Teletrece ${ }^{11}$ emitió en Facebook Live imágenes de un dron que seguía las celebraciones de los aficionados de "la Roja" en la Plaza Italia de Santiago. Este contenido obtuvo 463 mil reproducciones, 2,5 mil compartidos y 3 mil comentarios.

Días más tarde, específicamente el 10 de julio de 2016, Portugal sorprendió a Francia y levantó su primera Eurocopa en París. Teletrece optimizó recursos y para la emisión de su corresponsal en la capital gala no fue necesario ningún satélite, con su teléfono móvil entrevistó a los aficionados lusos a la salida del estadio e informó de las principales celebraciones. Con ello, se alcanzaron las 42 mil reproducciones, 92 compartidos y 262 comentarios. Todo ello fue destacado por el Facebook Journalism Project de la siguiente manera "Los resultados fueron sumamente positivos, ya que se recibió una gran cantidad de interacciones y preguntas, que resultaron útiles para guiar la futura programación con Live, y se realizaron publicaciones en el sitio web de Teletrece" (Facebook, s.f.).

\footnotetext{
${ }^{9}$ Noticiero emitido por Canal 13 de Chile. Ver más en http://www.t13.cl/

${ }^{10}$ El canal de Teletrece en Facebook https://www.facebook.com/Teletrece/ cuenta con más 3,6 millones de seguidores al 26/10/17.

${ }^{11}$ Los datos mencionados fueron extraídos el día 17/10/17. 
Figura 3: Principales resultados de Teletrece en Facebook Live

\section{Ejemplo caso Teletrece}

- Facebook Journalism Project lo destaca como caso de éxito en dos coberturas deportivas

- Final Eurocopa 2016: celebración hinchas portugueses en París

- Copa América Centenario: programa diario especial, conferencia de prensa de la Roja, y dron con celebraciones en Plaza Italia

\section{Resultados}

- Más de 530 mil reproducciones

- Más de 2.500 contenidos compartidos

- Cerca de 3.700 comentarios

Fuente: Elaboración propia.

Sin embargo, aún hay casos donde Periscope ofrece mejores resultados. Uno de ellos es el de Efecto Cocuyo de Venezuela que, sin grandes recursos, ya cuenta con cerca de mil videos en directo publicados ${ }^{12}$, los cuales son de tres tipos: sucesos de última hora, emisión de grandes eventos y un programa de estudios. Durante la Consulta Nacional de Venezuela realizada por su Asamblea Nacional el 16 de julio de 2017, Efecto Cocuyo realizó un total de 12 emisiones, todas ellas desde un único teléfono móvil. En total, completaron 82 minutos al aire y superaron los 53 mil espectadores un tercio de ellos durante la emisión en directo- cada uno de los cuales visualizó un promedio de 51 segundos de video. La integración de Periscope con Twitter les permitió duplicar el contenido en ambas redes, por lo cual las 12 emisiones se transformaron en 54 comentarios, 1.169 retweets y 586 likes (Apablaza-Campos A. , 2019, págs. 89-91).

Figura 4. Principales resultados de Efecto Cocuyo en Periscope

\section{Efecto Cocuyo y la Consulta Nacional de Venezuela}

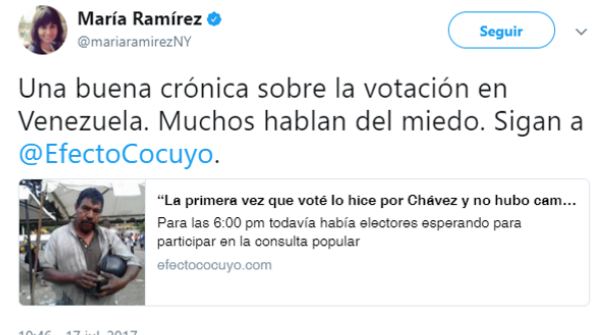

- 12 emisiones en Periscope

- 53,3k espectadores en total

- $17 \mathrm{k}$ reproducciones en directo

- 1.809 interacciones en Twitter

\section{Resultados analizados}

- 11 usuarios alcanzados por segundo emitido

- Cada espectador visualizó un promedio de 51 segundos

Fuente: Elaboración propia.

\footnotetext{
${ }^{12}$ El canal de Efecto Cocuyo en Periscope https://www.pscp.tv/EfectoCocuyo cuenta con cerca de 28 mil seguidores al 26/10/17. Revista Pensamiento Académico de la Universidad UNIACC 
En la vereda de Facebook Live han buscado una serie de argumentos para atraer a los medios digitales. Mientras la jefa de video de la red social, Fidji Simo, afirma que una de cada cinco reproducciones en Facebook corresponde al live streaming (Constine, 2017); Luis Renato Olivalves, director de relación con medios de Facebook en América Latina, sostiene que el volumen de comentarios es 10 veces mayor en un contenido en directo, y que el número de reproducciones es tres veces más. Anuncios que fueron estudiados en una comparativa realizada a través de los videos publicados en el canal de Facebook de NTN24 Venezuela ${ }^{13}$ durante las Elecciones para la Asamblea Nacional Constituyente realizada el 30 de julio de 2017.

En la Figura 5 es posible apreciar que, de las 35 piezas emitidas durante la jornada, efectivamente un quinto de ellas es en directo; que el volumen de comentarios es 30 veces mayor; y que, si bien las reproducciones en vivo no triplican a las convencionales, sí son 2,5 mayores. Incluso es posible ver cómo las reacciones ${ }^{14}$ son 4,6 mayores.

Figura 5: Comparativa entre videos convencionales y videos en directo emitidos por NTN24 Venezuela en Facebook el 30/07/17

\begin{tabular}{|c|c|c|c|c|c|c|}
\hline $\begin{array}{l}\text { TIPOS DE } \\
\text { CONTENIDOS }\end{array}$ & EMITIDOS & $\begin{array}{l}\text { DURACIÓN } \\
\text { PROMEDIO }\end{array}$ & $\begin{array}{l}\text { REPRODUCCIONES } \\
\text { PROMEDIO }\end{array}$ & $\begin{array}{l}\text { COMPARTIDOS } \\
\text { PROMEDIO }\end{array}$ & $\begin{array}{l}\text { COMENTARIOS } \\
\text { PROMEDIO }\end{array}$ & $\begin{array}{l}\text { REACCIONES } \\
\text { PROMEDIO }\end{array}$ \\
\hline $\begin{array}{l}\text { VIDEOS } \\
\text { CONVENCIONALES }\end{array}$ & 28 & 1:10 & 61.022 & 2.356 & 136 & 786 \\
\hline VIDEOS EN DIRECTO & 7 & $10: 40$ & 153.660 & 2.200 & 3.327 & 3.633 \\
\hline
\end{tabular}

\section{El caso de NTN24 Venezuela en las Elecciones Regionales 2017}

Por norma general, las jornadas electorales cuentan con amplia cobertura informativa. Los medios emplean sus recursos digitales para generar contenido continuo, y recursos como el Social Media Live Streaming permiten informar desde distintos lugares. NTN24 Venezuela no fue la excepción durante el 15 de octubre de 2017. Informaciones desde centros de votación, además conferencias de prensa de autoridades y partidos políticos tanto antes como durante y después de la elección fueron los principales contenidos emitidos a través del video en directo en redes sociales. En esta ocasión, se realizó un seguimiento a sus canales de Periscope ${ }^{15} \mathrm{y}$ Facebook Live. En el día se efectuaron un total de 24 videos en directo -14 en Periscope y 10 en Facebook Live-. Tal como se puede ver en la Figura 6, si bien el

\footnotetext{
${ }_{13}$ El canal de NTN24 Venezuela en Facebook https://www.facebook.com/NTN24ve/ cuenta con más de 1,6 millones de seguidores al 09/09/21.

${ }^{14}$ Facebook considera como "reacciones" a los clicks en las opciones “Me gusta”, “Me encanta”, “Me divierte”, “Me asombra", "Me entristece" y "Me enfada".

15 El canal de NTN24 Venezuela en Periscope https://www.pscp.tv/NTN24ve cuenta con más de 41 mil seguidores al o9/o9/21. Revista Pensamiento Académico de la Universidad UNIACC Vol. $4 \mathrm{~N}^{\circ} 1,2021$
} 
primer el primer canal social un mayor número de emisiones, el segundo obtuvo el doble de reproducciones.

Figura 6. Comparativa de contenidos emitidos por NTN24 Venezuela a través de Facebook Live y Periscope el $15 / 10 / 17$

\begin{tabular}{llllll} 
SMLS & EMITIDOS & $\begin{array}{l}\text { DURACIÓN } \\
\text { PROMEDIO } \\
\text { (MIN:SEG) }\end{array}$ & $\begin{array}{l}\text { DURACIÓN } \\
\text { TOTAL } \\
\text { (HRS:MIN:SEG) }\end{array}$ & $\begin{array}{l}\text { REPRODUCCIONES } \\
\text { PROMEDIO }\end{array}$ & $\begin{array}{l}\text { REPRODUCCIONES } \\
\text { TOTALES }\end{array}$ \\
PERISCOPE & 14 & $22: 33$ & $5: 15: 40$ & $11.528,21$ & 161.395 \\
FACEBOOK LIVE & 10 & $17: 40$ & $2: 28: 55$ & 28.574 & 342.016 \\
& \multicolumn{2}{r}{ Fuente: Elaboración propia. }
\end{tabular}

Sin embargo, es necesario realizar las siguientes consideraciones al respecto:

- Si una conclusión que permite la Figura 6 es que NTN24 Venezuela obtiene el doble de visualizaciones en Facebook Live emitiendo menos contenido que en Periscope, también es necesario tener en cuenta que el número de seguidores en el fanpage es 21 veces superior al de la red SMLS.

- Para realizar la Figura 6 se analizaron las estadísticas que entregó cada red a las 23:59 hora española del 16/10/2017. Solo se consideraron los datos que pudiesen tener puntos de comparación entre sí, debido a que Facebook entrega datos que Periscope no -reacciones, comentarios y compartidos- y viceversa -reproducciones en directo y reproducciones en repetición-.

- No es posible realizar una comparativa de resultados en simulcasting, es decir, de ambos canales reproduciendo el mismo contenido en simultáneo. Esto porque, del total de contenidos emitidos, solo cinco tienen temáticas coincidentes, pero no así la duración y los horarios de emisión.

- En materia de contenidos es importante destacar que todas las emisiones de Facebook Live de NTN24 Venezuela corresponden a redifusiones de la señal televisiva -emitida también a través de YouTube y de la web-, mientras que en Periscope es posible encontrar emisiones de sucesos de última hora e incluso retransmisiones desde otras cuentas de Periscope.

- En línea de lo anterior y en cuanto al tipo de contenidos emitidos, llama la atención que la última emisión del día en Facebook Live es a minutos del cierre de las mesas de votación. Todo el proceso de recuento de votos, información de resultados y reacciones de sectores políticos se emitió únicamente mediante Periscope.

- Periscope reemitió mensajes de líderes políticos que hablaban en la misma red, lo que pudo generar resultados más bajos que las emisiones habituales, considerando que muchos usuarios podían preferir ver el contenido directo desde el canal oficial. 
- Pese a que está la alternativa de replicar los contenidos de Periscope en Twitter automáticamente, NTN24 Venezuela no usó esa alternativa, pero sí compartió de forma manual la mitad de los contenidos generados.

- En resumen, es posible comprobar que NTN24 Venezuela implementó una estrategia de contenidos diferenciada para cada uno de sus canales de Social Media Live Streaming.

Figura 7. Modo de visualización de usuario SMLS en un contenido común (entrevista a un candidato a la salida del lugar de votación). Arriba en Facebook Live y abajo en Periscope.

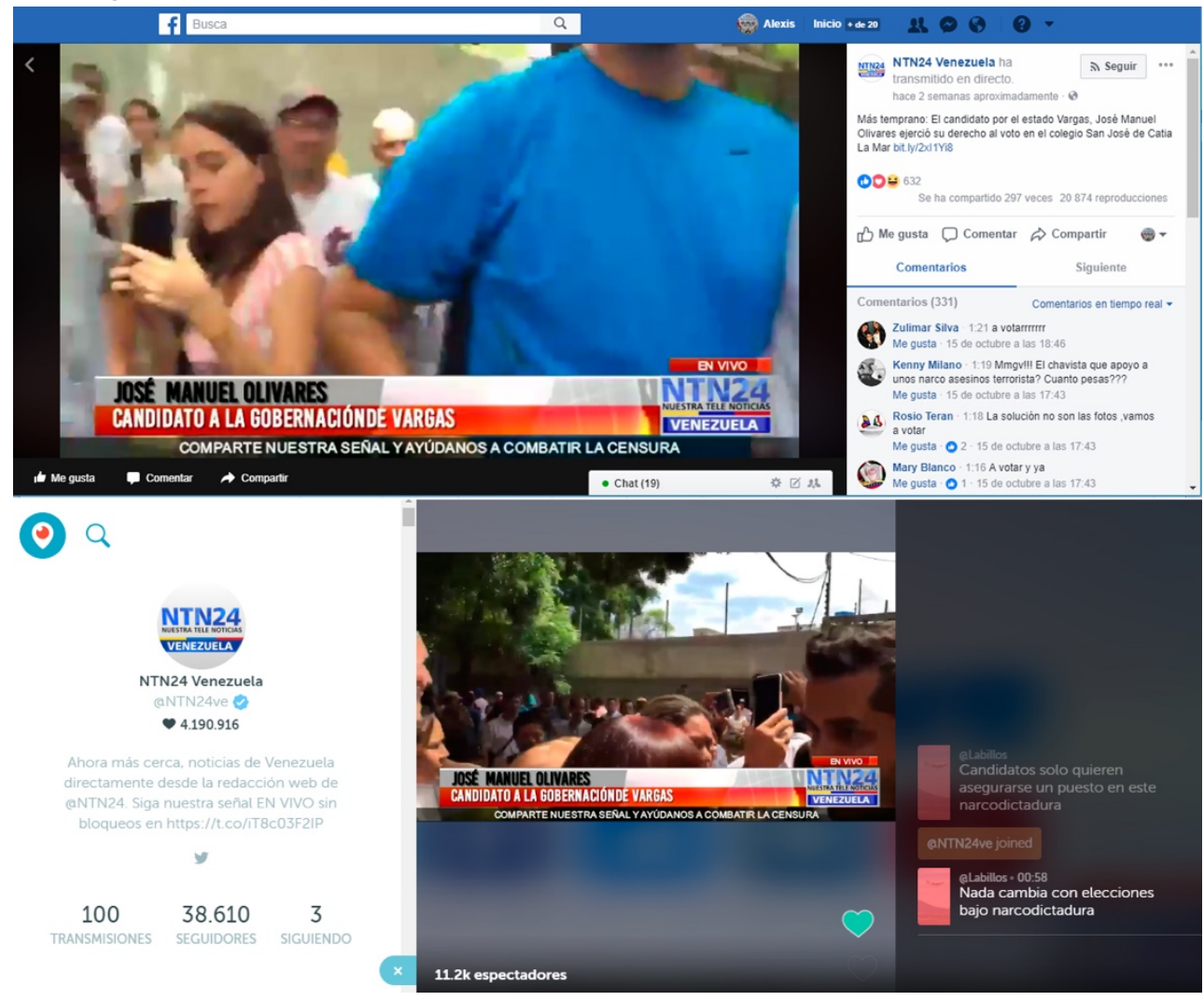

\section{Conclusiones}

1) El principio del siglo XXI trajo consigo una crisis tanto a internet como a todos los Entornos Digitales asociados, obligándolos a repensarse a sí mismos como método de existencia. Con ello se desarrollaron aplicaciones que revolucionaron el consumo de video en línea como YouTube y Netflix, aprovechando el crecimiento de las conexiones a internet en distintos dispositivos.

2) Pese al fracaso de algunas experiencias de videos en directo a través de la web o de aplicaciones móviles, el Social Media Live Streaming ha conseguido un crecimiento sostenido desde hace un par de años. Sus claves radican tanto en la emisión en diversos formatos a través de las redes sociales como en sus oportunidades de feedback continuo a sus audiencias. 
3) Es posible encontrar diversos programas que emplean las tecnologías SMLS, pero hasta el momento Periscope y Facebook Live son aquellos que están funcionando con mayor éxito, especialmente por su nivel de alcance a usuarios y por sus posibilidades de almacenamiento de contenidos.

4) Periscope tuvo un rápido crecimiento inicial, y actualmente está totalmente integrada a su matriz Twitter. Continuamente está buscando fórmulas para generar ingresos a los generadores de contenido, con recursos como emoticones pagados (Superhearts) o anuncios (pre-roll SMLS ADS).

5) El respaldo de Facebook ha sido el principal soporte que ha consolidado a Facebook Live a la cabeza de contenidos SMLS. Su fundador ha insistido en que el video en línea es cada vez más importante en la navegación.

6) Las proyecciones acerca de la importancia del SMLS para los medios digitales y sus coberturas informativas se basan en las experiencias exitosas previas de editores de todo el mundo.

7) En cuestión de meses las redacciones se han adaptado al empleo del live streaming como un recurso para mejorar la visibilidad de sus coberturas informativas. El uso espontáneo de un periodista en el lugar de los hechos ha sido reemplazado por decisiones integradas en las estrategias digitales.

8) Los editores latinoamericanos no han estado ajenos a este fenómeno, y en gran parte de los medios digitales de la región ya cuentan con una serie de experiencias en SMLS suficientes como para saber qué pueden hacer para mejorar en la siguiente transmisión. Incluso, muchos de ellos han realizado emisiones experimentales en diferentes plataformas, lo cual les permite saber qué red les entrega mejores resultados.

9) Existe un caso de coberturas informativas a través de SMLS de un medio latinoamericano destacado a nivel mundial. Se trata del noticiero chileno Teletrece que aprovechó los derechos de transmisión de grandes eventos deportivos para ofrecer contenido exclusivo, y alternativo a la emisión de los partidos, a sus seguidores en Facebook Live.

10) Periscope permite diferenciar resultados entre reproducciones en directo y reproducciones en retransmisión. La opción de generar contenido en simulcasting con Twitter permite incluir las analíticas de la red de mensajes breves al análisis de resultados.

11) Con Facebook Live es posible realizar una comparativa de los resultados de los videos en directo con los videos convencionales en un fanpage. En los casos estudiados, es correcto sostener que el live streaming entrega más oportunidades de conectar con las audiencias.

12) Gracias a las estadísticas entregadas por Periscope y Facebook Live es posible realizar una comparativa de resultados entre diferentes cuentas. 
13) Finalmente, y en línea con el punto anterior, es importante destacar la complejidad de establecer un análisis comparativo con exactitud para saber qué canal entrega mejores resultados ante la difusión de un contenido específico. Además, por norma general, los medios digitales que emiten contenidos en diversos canales SMLS ofrecen estrategias diferenciadas en cada perfil.

\section{Referencias}

Alexa. (2017). The top 500 sites on the web. http://www.alexa.com/topsites

Apablaza Campos, A. (2017). Periscope y la videorealidad periodística. I Congreso Internacional de Periodismo: Convergencias Mediáticas y Nueva Narrativa Latinoamericana (pp. 141-153). Ciespal. http://ciespal.org/wpcontent/uploads/2015/10/ACTAS-Periodismo-FINAL.pdf

Apablaza-Campos, A. (2018a). Social media live streaming (SMLS) in the digital news media. Comunicació: Revista de Recerca i d'Anàlisi, 103-123.

Apablaza-Campos, A. (2019). Social Media Live Streaming (SMLS) para medios digitales: características, análisis comparativo y estudios de casos. Tesis Doctorals en Xarxa. http://hdl.handle.net/10803/667525

Apablaza-Campos, A., \& Codina, L. (2018b). Social Media Live Streaming: estudio de caso y diseño de matriz de análisis. Cuadernos.info(43), 161-180.

Codina, L., Iglesias García, M., Pedraza, R., \& García Carretero, L. (2016). Visibilidad y posicionamiento web de informaciones periodísticas:/nel framework SEORCP. e-Repositori UPF.

Columbia Journalism Review. (2015). Hard numbers: The new live streaming. CJR.

comScore. (2015). Chile Digital Future in Focus.

comScore. (2016). GLOBAL DIGITAL FUTURE IN FOCUS.

Constine, J. (2017, Abril 06). One in five Facebook videos is Live as it seizes the verb. https://techcrunch.com/2017/04/06/live-video/

Dircomfidencial. (2020, enero 22). Netflix eleva un 54\% su beneficio en 2019 pero se frena el aumento de suscriptores en EEUU. https://dircomfidencial.com/medios/netflix-eleva-un-54-su-beneficio-en-2019pero-se-frena-el-aumento-de-suscriptores-en-eeuu-20200122-0403/ 
Doctor, K. (2010). Newsonomics: Twelve New Trends That Will Shape the News You Get. St. Martin's Press.

El Economista América. (2016, enero 5). Periscope: la aplicación estrella del año 2015. http://www.eleconomista.es/telecomunicacion-tecnologiacl/noticias/7259884/01/16/-Periscope-la-aplicacion-estrella-del-ano-2015.html

El Universal. (2014, febrero 13). Maduro: Sacar del aire a NTN24 fue una decisión de Estado. http://www.eluniversal.com/nacional-y-politica/140213/maduro-sacardel-aire-a-ntn24-fue-una-decision-de-estado

Facebook. (2017, agosto 10). AS Roma celebra un hito junto a hinchas de todo el mundo mediante Facebook Live. https://www.facebook.com/facebookmedia/successstories/asroma

Facebook. (n.d.). Editores de noticias de todo el mundo triunfan con Facebook Live. Facebook Media. https://www.facebook.com/facebookmedia/successstories/globalnews-live

Facebook Media Blog. (2017, 5 de enero). How The Associated Press Trains its Journalists To Shoot in 360 Degrees. https://media.fb.com/2017/01/05/how-theassociated-press-trains-its-journalists-to-shoot-in-360-degrees/

Facebook Media. (s/f). Jorge Ramos cubre las elecciones en Estados Unidos con Facebook Live. https://www.facebook.com/facebookmedia/successstories/jorge-ramos

Fairweather, T. (2017, 10 de febrero). 6 tips to prepare your newsroom for live video on social media. Journalism.co.uk: https://www.journalism.co.uk/skills/6-tips-toprepare-your-newsroom-for-live-video-on-social-media/s7/a699561/

Faklaris, C., Cafaro, F., Anne Hook, S., Blevins, A., O’Haver, M., \& Singhal, N. (2016). Legal and Ethical Implications of Mobile Live-Streaming Video Apps. 18th International Conference on Human-Computer Interaction with Mobile Devices and Services. MobileHCl.

Flipboard. (n.d.). Social Media Live Streaming. https://flipboard.com/@alexisapabl6gtn/social-media-live-streamingtggabbcfz

Fundación Gabriel García Márquez para el Nuevo Periodismo Iberoamericano. (2016). Ocho claves para entender las audiencias digitales en Colombia. FNPI. 
García Carretero, L., Codina, L., \& Pedraza, R. (2016). Indicadores para el estudio de la visibilidad y del impacto de los cibermedios en el ecosistema digital. eRepositori UPF.

Gomis, L. (1991). Teoría del periodismo: cómo se forma el presente. Paidós.

Gupta, C. (2017, julio 25). Introducing the Live 360 Ready Program and new features for Live 360. Facebook Media. https://media.fb.com/2017/07/25/introducing-thelive-360-ready-program-and-new-features-for-live-360/

Hern, A. (2017, 5 de enero). Facebook Live is changing the world - but not in the way it hoped. The Guardian.

https://www.theguardian.com/technology/2017/jan/05/facebook-live-socialmedia-live-streaming

Herrero de la Fuente, M. (2017). Nuevas fórmulas para la televisión en directo: el uso de Facebook Live en Atresmedia. Miguel Hernández Communication Journal, 521-563.

IMS; comScore. (2016). IMS Mobile in Latam Study . Miami FL, USA: IMS Corporation.

Ingram, M. (2016, 2 de marzo). Facebook May be Playing Catch-Up on Video, but It Is Going All In. Fortune: http://fortune.com/2016/03/02/facebook-video/

Instagram. (2020, 17 de abril). Cómo iniciar y organizar un Instagram Live . Instagram for Business. https://business.instagram.com/blog/instagram-livelatam?locale $=$ es_LA

Kalogeropoulos, A., Cherubini, F., \& Newman, N.(2016). Digital News Proyect. Reuters Institute.

Knight Center for Journalism in the Americas. (2017). Periodismo Innovador en América Latina. (T. Mioli, \& I. Nafría, eds.) Open Society Foundations.

Lahiri, S., \& Tiutan, C. (2017, Septiembre 13). Introducing Updates to the Live API. Facebook Media. https://media.fb.com/2017/0g/13/introducing-updates-tothe-live-api/

Linares, J., Codina, L., Vállez, M., \& Rodríguez Martínez, R. (2016). Interactividad, buscabilidad y visibilidad en cibermedios: sistema de análisis y resultados. $e$ Repositori UPF.

LinkedIn. (s.f.). LinkedIn Live Video. Ayuda LinkedIn. https://www.linkedin.com/help/linkedin/answer/100224 
López, N. (2017, Octubre 23). Facebook tests Live Video Producer Tool with multicamera support and GFX features. The Next Web: https://thenextweb.com/facebook/2017/10/23/facebook-tests-live-videoproducer-for-advanced-streaming-tools/

Mancebo García, M. (2016). Innovación en formatos audiovisuales. El uso de herramientas de live streaming en Antena 3 y Univision. Miguel Hernández Communication Journal .

Mlot, S. (2016, Octubre 3). Streaming App Meerkat Is Officially Dead. Retrieved from PC Mag: https://www.pcmag.com/news/348393/streaming-app-meerkat-isofficially-dead

Nación. (2006, Noviembre 26). 'Time' escoge a You Tube como el invento del 2006. http://www.nacion.com/vivir/Time-escoge-You-Tubeinvento_o_869313124.html

Nazario, J. (2017, 27 de abril). ISOJ 2017: Panelistas ven éxitos y limitaciones de formatos innovadores de video, incluidos Facebook Live y realidad virtual. Knight Center of Journalism in the Americas. https://knightcenter.utexas.edu/es/blog/oo18320-isoj-2017-panelistas-ven-exitos-y-limitaciones-de-formatosinnovadores-de-video-inclui

Nelson, J. (2017, 10 de enero). What is a Facebook Live editor? (R. J. Institute, Editor, \& University of Missouri). https://www.rjionline.org/stories/what-is-a-facebooklive-editor

Newman, N. (2017a). Digital News Project 2017. Oxford: Reuters Institue \& University of Oxford.

http://reutersinstitute.politics.ox.ac.uk/sites/default/files/Journalism\%2C\%2OM edia\%20and\%20Technology\%20Trends\%20and\%20Predictions\%202017.pdf

Newman, N., Fletcher, R., Kalogeropoulos, A., Levy, D., \& Kleis Nielsen, R. (2017b). Digital News Report 2017. Reuters Institue \& University of Oxford.

Ossorio Vega, M. Á. (2015, 20de junio). Periscope, el vídeo en directo para todos. (Universidad Complutense de Madrid). Internet Media Lab. http://internetmedialab.com/2015/06/30/periscope-el-video-en-directo-paratodos/

Palacios, M., \& Díaz Noci, J. (2007). Ciberperiodismo: Métodos de Investigación. Servicio Editorial de la Universidad del País Vasco.

Pastor, J. (2017, 20 de junio). Las nuevas gafas de Hawkers integran una cámara y hacen livestreaming vía Periscope. Xataka. https://www.xataka.com/accesorios/lasRevista Pensamiento Académico de la Universidad UNIACC 
nuevas-gafas-de-hawkers-integran-una-camara-y-hacen-livestreaming-viaperiscope

Periscope. (2020, diciembre 15). Farewell, Periscope. Medium. https://periscope.medium.com/farewell-periscope-164db2742b7c

Perlberg, S., \& Sheetharaman, D. (2016, 22 de junio). Facebook Signs Deals With Media Companies, Celebrities for Facebook Live. The Wall Street Journal. https://www.wsj.com/articles/facebook-signs-deals-with-media-companiescelebrities-for-facebook-live-1466533472

Pew Research Center. (2015). The Evolving Role of News on Twitter and Facebook. Pew Research Center.

Pichihua, S. (2017, 16 de julio). El video en Facebook Live nominado a los Emmy. http://www.clasesdeperiodismo.com/2017/07/16/el-video-en-facebook-livenominado-a-los-emmy/

Publimetro Chile. (2016, 6 de enero). Conoce Periscope: la aplicación que se consolidó durante el 2015. https://www.publimetro.cl/cl/teknik/2016/01/06/conoceperiscope-aplicacion-que-se-consolido-durante-2015.html

Reuters Institute. (2017). Digital News Report 2017 . University of Oxford.

Rugg, A., \& Burroughs, B. (2016). PERISCOPE, LIVE-STREAMING AND MOBILE VIDEO CULTURE. Geoblocking and Global Video Culture, 64-73.

Salza, C. (2017, Mayo 8). Facebook lanzará programas de TV propios en junio: reporte. CNET. https://www.cnet.com/es/noticias/facebook-lanzara-programas-detelevision-propios-en-junio/

Scolari, C. (Ed.). (2015). Ecología de los Medios: Entornos, evoluciones e interpretaciones. Gedisa.

SEO Media Lab. (n.d.). Estudios de visibilidad. Medios y sectores económicos: https://seomedia-lab.com/

Seymour, D., \& Smekalina, J. (2017, Julio 11). The Hill Goes Live and Raises Money During the Congressional Baseball and Softball Games. Facebook Media. https://media.fb.com/2017/07/11/the-hill-goes-live-and-raises-money-duringthe-congressional-baseball-games/

Shawley, P. (2016). A fresh face for storytelling. Television, 46-47. https://rts.org.uk/sites/default/files/new_television-oct16-lo-res.pdf 
Stewart, D. R., \& Littau, J. (2016). Up, Periscope: Mobile Streaming Video Technologies, Privacy in Public, and the Right to Record. JOURNALISM \& MASS COMMUNICATION QUARTERLY, 312-323.

Strange, A. (2017, julio 12). Facebook Spaces adds live video streaming, letting people with or without Oculus Rift headsets participate in social VR. Mashable. http://mashable.com/2017/07/12/facebook-space-live-streaming/?utm_cid=hpr-13\#b1NzCZNGSiqq

Stulberg, A. (2017, 22 de septiembre). In paywall age, free content remains king for newspaper sites. Columbia Journalism Review. https://www.cjr.org/business_of_news/newspaper-paywalls.php

Thompson Reuters. (2017, 19 de octubre). Reuters launches next generation live video service for news broadcasters and digital publishers on Reuters Connect platform. https://www.thomsonreuters.com/en/pressreleases/2017/october/reuters-launches-next-generation-live-video-servicefor-news-broadcasters-and-digital-publishers.html

TikTok. (s.f.). ¿Qué es TikTok LIVE?. Centro de Ayuda de TikTok. https://support.tiktok.com/es/live-gifts-wallet/tiktok-live/what-is-tiktok-live

Twitter. (s.f.). Preguntas frecuentes sobre Periscope. Centro de ayuda de Twitter: https://help.twitter.com/es/using-twitter/periscope-faq

Universitat Pompeu Fabra. (n.d.). Observatorio de Cibermedios. OCM. https://observatoriocibermedios.upf.edu/

Villanueva Baselga, S. (s/f). CINE Y CAMBIO SOCIAL Análisis y caracterización del vídeo participativo como objeto documental. Universidad de Barcelona.

Xinhua. (2017, 28 de octubre). China tiene 950 millones de usuarios de 4G. http://spanish.xinhuanet.com/2017-10/28/c_136710825.htm

YouTube. (s.f.). Introducción a las emisiones en directo. https://support.google.com/youtube/answer/2474026?co=GENIE.Platform\%3 DAndroid\&hl=es 


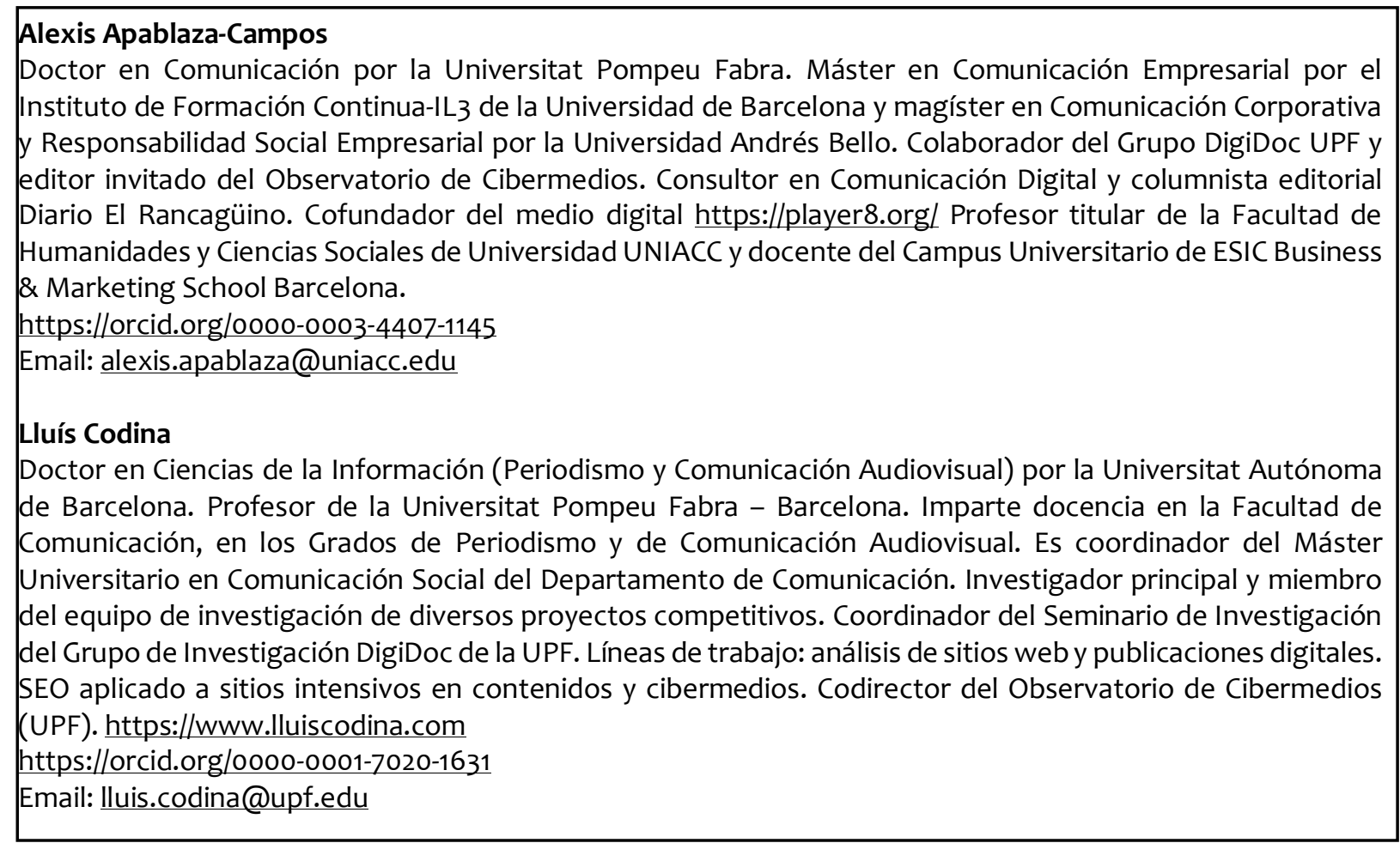

